\title{
Staff Mental Health Self-Assessment During the COVID-19 Outbreak
}

With the COVID-19 outbreak, vigilance in infection control has increased in both general public and hospital levels, and its psychological impact on hospital staff is expected to increase. In a survey in Canada about the psychosocial effects of Severe Acute Respiratory Syndrome on hospital staff, $29 \%$ of the respondents scored above the threshold point on the 12-item General Health Questionnaire, indicating probable emotional distress. ${ }^{1}$ Frontline healthcare workers may experience fear of being infected and spreading the virus to their families, particularly those working in isolation wards and accident and emergency departments. There is a need for timely mental health care for patients and health workers during the COVID-19 outbreak. ${ }^{2}$

With the high anticipated stress among hospital staff in Hong Kong East Cluster, a comprehensive support programme named Support of You (SOY) is initiated. Since 14 February 2020, the Department of Psychiatry of Pamela Youde Nethersole Eastern Hospital has provided an online mental health self-assessment questionnaire to all hospital staff in the cluster. It consists of the Patient Health Questionnaire-9 (PHQ-9), a free-text response, and an anonymous way to request for psychological support. An experienced advanced practice psychiatric nurse with training in psychotherapy was appointed to contact staff who needed help. The online questionnaire was distributed through the COVID-19 newsletter via email and hospital smartphone app, together with other mental health information and hospital resources for psychological support.

Of 8418 full-time equivalent staff in 6 hospitals, 69 staff have responded within 10 days of launching the online survey, including nurses $(\mathrm{n}=24,34.8 \%)$, clerical and administrative staff $(\mathrm{n}=23,33.3 \%)$, healthcare assistants $(\mathrm{n}=8,11.6 \%)$, and allied health staff $(\mathrm{n}=6,8.7 \%)$, doctors $(\mathrm{n}=3,4.3 \%)$, management staff $(\mathrm{n}=2,2.9 \%)$, and others $(\mathrm{n}=3,4.3 \%)$. The mean PHQ-9 score was 7.6, with 24 (34.8\%) respondents having a score of $\geq 10$ (the cut-off for mild depression) and $10(14.5 \%)$ respondents having a score of $\geq 15$ (the cut-off for moderate depression). Two (2.9\%) respondents requested support and were reassured after a single phone contact by the psychiatric nurse. 31 (44.9\%) respondents provided free-text about their concerns, with 14 (45.2\%) about the sufficiency of personal protective equipment and $6(19.6 \%)$ about being infected with COVID-19.

Although only a small proportion of hospital staff have responded, they are more likely to be psychologically affected by the outbreak. Nonetheless, there is no new case of psychological distress caused by the COVID-19 outbreak in the Critical Incident Psychological Services of the Centre for Personal Growth and Crisis Intervention in Hong Kong East Cluster. Staff may have concern about seeking help from hospital psychological service, and an anonymous online assessment provides a good platform for staff to assess their own mental well-being and to seek help.
Nurses have frequent contact with patients and were most ready to respond. Clerical and administrative staff were also readily responded despite their non-frontline, non-patient-care job nature. Comprehensive survey about the psychological effect of the COVID-19 outbreak on hospital staff of different ranks and positions is needed to provide timely and appropriate interventions. In China, a national guideline has been established for emergency psychological crisis intervention for the COVID-19 outbreak, and it recommends that (1) frontline staff involving in the care of COVID-19-infected patients should receive prior psychological crisis intervention trainings to anticipate patient's psychological reactions; (2) staff working in isolation ward should be put on rotations; (3) accommodation should be provided to frontline staff for self-isolation; (4) hotline and online psychological crisis interventions should be provided to needed staff; (5) and a psychological response team consisting of psychiatrists, psychologists, and psychiatric nurses should be formed in each unit to provide staff with psychological support. ${ }^{3}$ Enhancing the psychological well-being of hospital staff during the COVID-19 outbreak is equally important to the fight against the outbreak.

\section{Declaration}

The authors have no conflict of interest to disclose.

Joseph PY Chung, MRCPsych, FHKCPsych, FHKAM(Psychiatry), Department of Psychiatry, Pamela Youde Nethersole Eastern Hospital, Hong Kong

Wai-song Yeung, MRCPsych, FHKCPsych, FHKAM(Psychiatry), Department of Psychiatry, Pamela Youde Nethersole Eastern Hospital, Hong Kong

Address for correspondence: Dr Joseph PY Chung, Department of Psychiatry, Pamela Youde Nethersole Eastern Hospital, 3 Lok Man Road, Chai Wan, Hong Kong. Email: chungpy2@ha.org.hk

Submitted: 25 February 2020; Accepted: 27 February 2020

\section{References}

1. Nickell LA, Crighton EJ, Tracy CS, Al-Enazy H, Bolaji Y, Hanjrah S, et al. Psychosocial effects of SARS on hospital staff: survey of a large tertiary care institution. CMAJ 2004;170:793-8. Crossref

2. Xiang YT, Yang Y, Li W, Zhang L, Zhang Q, Cheung T, et al. Timely mental health care for the 2019 novel coronavirus outbreak is urgently needed. Lancet Psychiatry 2020;7:228-9. Crossref

3. National Health Commission of China. Principles for emergency psychological crisis intervention for the new coronavirus pneumonia [in Chinese]. Available at http://www.nhc.gov.cn/jkj/s3577/202001/6 adc08b966594253b2b791be5c3b9467.shtml. Accessed 24 February 2020 . 